Kaur Alttoa

\title{
DIE ST. KATHARINEN-KAPELLE IN FELLIN (VILJANDI)
}

In der mittelalterlichen Sakrallandschaft Estlands verfügten auch stadtnahe Kapellen über einen sicheren Platz. So gab es in Reval (estn. Tallinn) Antonius-, Barbara und Gertrud-Kapellen und die Kapelle des Johannes-Spitals. Aus Dorpat (estn. Tartu) sind außerhalb der Stadtmauern die Anna- und die Antonius-Kapelle bekannt sowie die St. Georg-Spitalskapelle. Mehrere Kapellen haben sich auch in der unmittelbaren Umgebung von Fellin befunden. Laut Information der Polen von 1599, welche die Stadt einer Revision unterzogen, waren hier früher die Jerusalem-, die Antonius-, die Elisabeth- und die St. Katharinen-Kapelle gewesen. ${ }^{1}$ Für die Mehrzahl begrenzt sich die gesamte Information hierauf und es fehlen jegliche weitere Hinweise über die Kapellen und ihre ungefähren Standorte. ${ }^{2}$ Ein wenig mehr wissen wir über die im Jahr 1506 erstmals erwähnte ${ }^{3}$ St. Katharinen-Kapelle. An dieser Stelle ist die von dem in Fellin aktiven Jesuitenpriester Dionysios Fabricius im zweiten Jahrzehnt des 17. Jahrhunderts verfasste Chronik die hauptsäch-

DOI: http://dx.doi.org/10.12697/BJAH.2015.10.06

Übersetzung aus dem Estnischen von Marju und Olaf Mertelsmann.

Einen Anstoß, den vorliegenden Beitrag zu verfassen, lieferte das auf Initiative von Prof. Christofer Hermann erfolgte Arbeitstreffen im Juni 2015 in Riga, in dessen Rahmen wir zusammen mit deutschen, polnischen und lettischen Kollegen auch in Cēsis (dt. Wenden) die St. Johanniskirche (lett. Jāṇa baznīza) besuchten. Dabei stieg erneut die Frage nach der Herkunft des Dekors der Kämpfer am Westportal der Kirche auf.

1 Polska XVI wieku pod wzgle,dem geograficzno-statystycznym. T. XIII, Inflanty, Cz. 1, hrsg. von Jan Jakubowski, Joźef Kordzikowski (Warschau: Gbethner i Wolff, 1915), 174.

2 Zur Antonius-Kapelle siehe: Kaur Alttoa, "Kahest Antoniuse kabelist ja ühest sealoost”, Kleio. 2 (Tartu, 1989), 23-24.

3 Est- und Livländische Brieflade. Erster Theil. Dänische und Ordenszeit, hrsg. von Friedrich Georg von Bunge, Robert von Toll (Reval: Kluge und Stroehm, 1856), Nr. 688. 


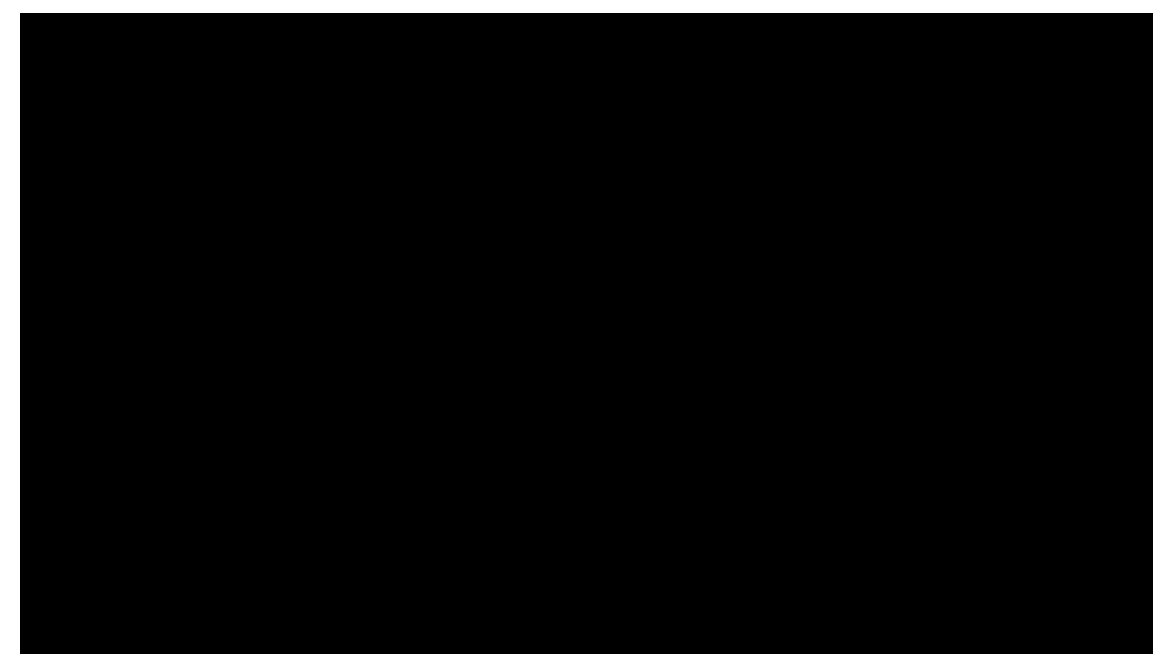

Abb. 1. Der ehemalige Standort der St. Katharinen-Kapelle in Viljandi.

liche Informationsquelle. Nach ihr war in Fellin "unterhalb der Festung eine sehr schöne Kapelle, geweiht der Heiligen Katharina und errichtet als Beerdigungsstätte für Träger des Kreuzes. Als der Moskowiter Narwa (estn. Narva) und Dorpat besetzte, wurde sie abgerissen auf Befehl von Fürstenberg, der fürchtete sie könnte der Festung gefährlich werden." ${ }^{\prime 4}$ Dieselbe Kapelle, wohl ohne die namensgebende Heilige zu erwähnen, beschreiben auch die 1599 in Fellin tätigen Revisoren: "eine Kirche war auch unterhalb der Festung, in der Nähe des Gartens, davon sind nur noch Spuren übrig geblieben. Dort beerdigt man Katholiken, dort sind auch die Leichen vieler bedeutender Menschen, dies bedeutet einige Ordensmeister und einige Dutzend bedeutendere polnische Soldaten." ${ }^{5}$ Die St. Katharinen-Kapelle wurde im Jahr 1558 abgerissen, doch Dionysius Fabricius stellt diesbezüglich noch einen Epilog vor. Nämlich soll ein Bürger Fellins sich aus den Steinen der abgerissenen Kapelle für sich ein Steinhaus errichtet haben. Danach begann das Sehvermögen des Hausbesitzers und seiner Gattin schwächer zu werden und am Ende erblindeten sie beide. Das Haus wurde verkauft, doch der nächste Eigentümer erblindete ebenfalls. ${ }^{6}$ Diese Geschichte ist

4 Dionysii Fabricii Praepositi pontificii Feliensis Livonicae historiae compendiosa series. Dionysius Fabriciuse, Viljandi kiriku praosti Liivimaa ajaloo lühiülevaade (Tartu: Johannes Esto Ühing, 2010), 192.

5 Polska XVI wieku, 174.

6 Dionysii Fabricii, 192. 


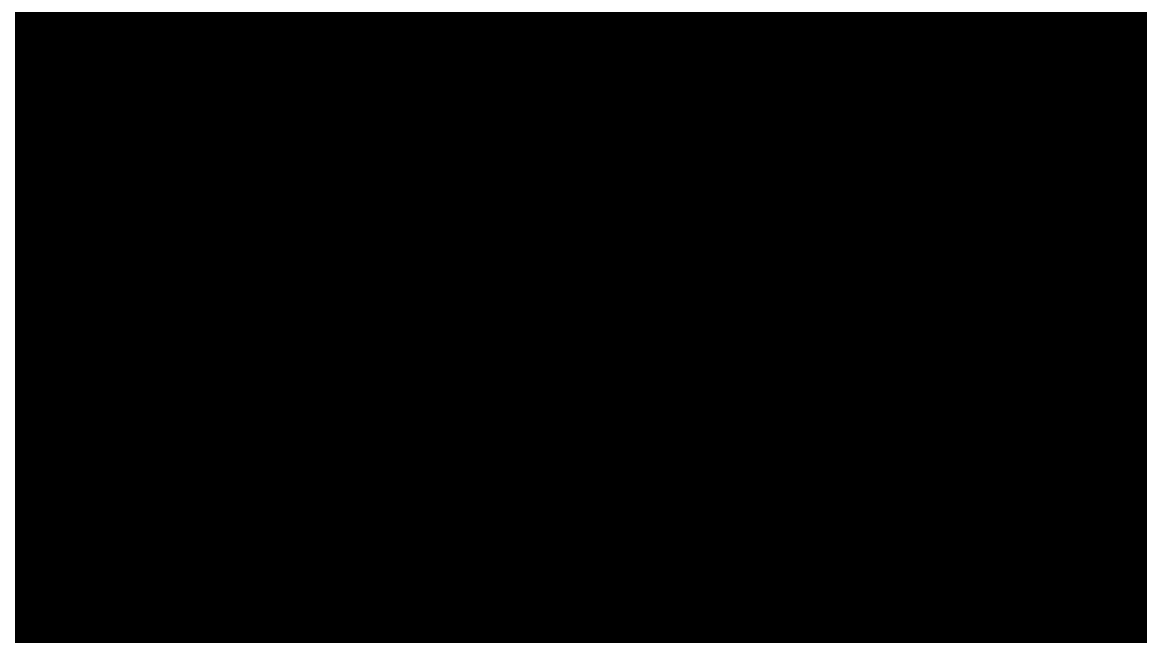

Abb. 2. Der Grundriss der St. Katharinen-Kapelle in Viljandi. Karl vom Löwis of Menar.

spannend und dramatisch, doch der Historiker befindet sich in einer gewissen Verwirrung. Nämlich haben die in Fellin 1599 aktiven, polnischen Revisoren exakt dieselbe Geschichte vom Erblinden präsentiert. Nur dass sich diese Erzählung auf die Jerusalem-Kapelle bezog. ${ }^{7}$

\section{DIE WIEDERÖFFNUNG DER KAPELLE IM JAHR 1908}

Von der St. Katharinen-Kapelle kann man heute keinerlei Spuren in Viljandi sehen. Doch ihr ehemaliger Standort ist leicht zu finden. Nämlich befindet sich unmittelbar hinter der Sängerbühne von Viljandi ein kleiner Hügel, auf dem eine Stützmauer aus Feldsteinen, eine Treppe mit Granitstufen sowie ein geschmiedetes Tor im Jugenstil zu sehen sind. Dies sind Anzeichen des ehemaligen Familienfriedhofs der Besitzer des Gutes Fellin, der von Ungern-Sternbergs. Vor einiger Zeit wurde dort auch ein Gedenkstein errichtet. Gerade die Einrichtung des Friedhofes 1907 ist verbunden mit der Wiedereröffnung der St. Katharinen-Kapelle. Denn man geriet damals auf die mittelalterlichen Mauern. Deshalb wurden hier im folgenden Jahr archäologische Ausgrabungen unter Leitung des Architekturhistorikers Karl von Löwis of Menar organi-

$7 \quad$ Polska XVI wieku, 174. 
siert $^{8}$ und ans Tageslicht kamen die Mauern der Kapelle, die ungefähr bis zu einer Höhe von einem Meter erhalten waren. Dieses Gebäude mit den Ausmaßen einer ordentlichen Kirchspielkirche bestand aus einem Langhaus (Innenmaße 14,5-15,5 x 12,2 m), das sich einem quadratischen Grundriss annäherte, und einem Chorraum. Beide waren ins Auge stechend asymmetrisch. Bei der Ausgrabung wurden zahlreiche Rippensteine aus Ton gefunden', damit verfügte die Kapelle über ein Gewölbe. Aufgrund der Raumproportionen kann vermutet werden, dass das Langhaus ein zweischiffiger Raum mit einem Zentralpfeiler war. Irgendwelche Spuren von dem Pfeiler wurden nicht gefunden, wahrscheinlich wurden sie im 18. Jahrhundert vernichtet, als auf dem Ort der Ruinen der Kapelle ein hölzernes Gutsgebäude errichtet wurde. ${ }^{10}$

Entsprechend dem zeitgenössischen Verständnis behandelt Karl von Löwis of Menar die Kapelle als innerhalb einer Bauperiode fertiggestellte. Irgendwelche Bemerkungen über verschiedene Bauabschnitte oder darauf hinweisende Fugen treffen wir nicht an. Möglich, dass es sie nicht gab. Dennoch treten einige Fragen auf. Auffallend ist der Unterschied der von Ost nach West gerichteten Achse von Langhaus und Altarraum: der letztere ist deutlich mehr nach Süden ausgerichtet. So manches Mal weist eine derartige Abweichung darauf hin, dass es sich um zu verschiedenen Zeiten errichtete Bauteile handelte. Als eigenartig erscheint auch die Wand zwischen Langhaus und Chor. Wenn die Mauern der Kapelle an anderer Stelle 1,50 bis 1,65 m dick sind, dann ist diese Wand sowohl laut Beschreibung ${ }^{11}$ als auch laut der Zeichnung nur $70 \mathrm{~cm}$ dick. Es bleibt unverständlich, wie eine solch dünne Mauer an einem Ort, wo sich ein 4,70 m breiter Triumphbogen befand, die Last des Gewölbes zu tragen vermochte. Notwendigerweise tritt nun die Frage danach auf, mit welcher Exaktheit der Architekt die Bausubstanz fixierte und interpretierte. Weiterhin hat Karl von Löwis of Menar auf Grundlage der sich in der Mitte der Ostwand befindlichen Wandvorlage behauptet, dass auch der Chor mit zwei Gewölben versehen war. Eine derartige Lösung wäre allerdings

8 Karl von Löwis of Menar, "Freilegung der Überbleibsel der St. Katharinenkapelle in Fellin", Jahresbericht der Felliner literarischen Gesellschaft für die Jahre 1907-1908. Beilage (Fellin: F. Feldt, 1909).

9 Sie befinden sich im Museum in Viljandi.

10 Frühere Forscher haben behauptet, dass der Gutshof im 17. Jahrhundert gebaut wurde. Offenbar ist dies dennoch erst nach dem Nordischen Krieg erfolgt. (Jekaterina Lissitsina, Aivar Kriiska, Kaur Alttoa, Leho Ainsaar, Alexander Kulkov, "Archaeological fieldwork during the reconstruction of the Viljandi song festival grounds", Archaeological Fieldwork in Estonia 2013 (Tallinn, 2014), 104).

11 Löwis of Menar, "Freilegung der Überbleibsel der St. Katharinenkapelle in Fellin", 6. 


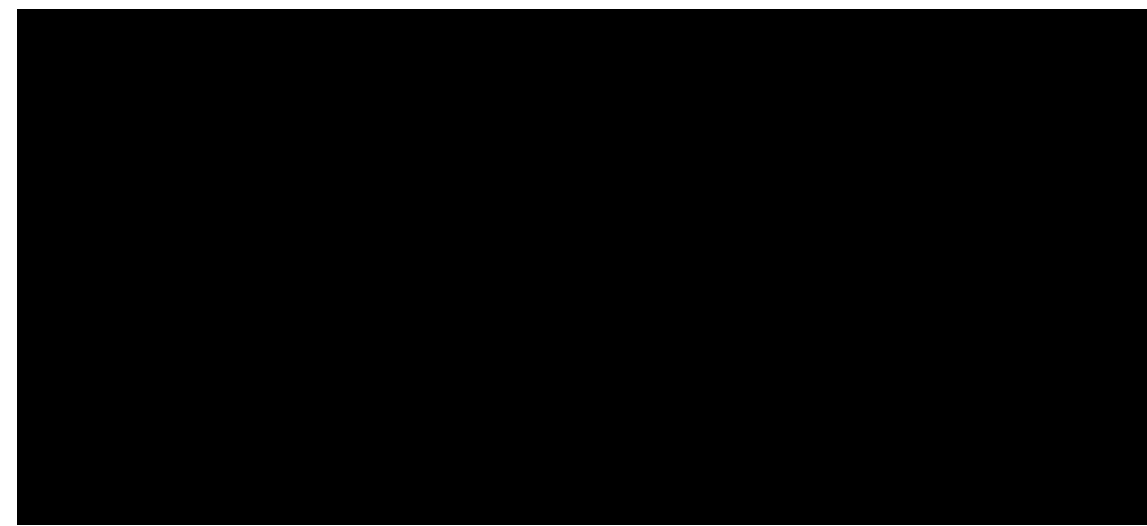

Abb. 3. Das Kapitell mit den Reliefs, entdeckt auf dem Standort der Kapelle. Foto von Kaur Alttoa.

ohne Beispiel. So stellt sich nötigerweise die Frage, ob das erwähnte Detail mit dem Grundmauerwerk verbunden war oder handelte es sich dennoch um eine sekundäre Beifügung. Auf jeden Fall fehlten im Langhaus jegliche Wandvorlagen zur Unterstützung von Gewölben.

\section{KAPITELL MIT FABELTIEREN, IHRE ANALOGIEN UND DIE DATIERUNGEN}

Der wichtigste Fund bei den Ausgrabungen war ein aus Kalkstein geschnittenes, achteckiges ${ }^{12}$ Kapitell, das sich heute im Museum in Viljandi befindet. Auf seinen Seiten waren ein Adler, Fabeltiere ebenso wie Pflanzenmotive, Weinlaub, Eichenlaub und eine Lilie, abgebildet. Ein Teil von ihnen befindet sich in einem herzförmigen Rahmen. Einzigartig ist die Handschrift des Bildhauers. Die ausgesprochen flachen Figuren sind nicht modelliert sondern streng in zwei Flächen gehalten, sie erinnern an Pfefferkuchen, die aus einem sehr dünnen Teig ausgeschnitten wurden. Für diese Manier wird auch der Terminus "Herbariumstil" verwendet. An einigen Orten sind auch das Federkleid des Vogels oder das Auge, der Schnabel oder die Ohren einer Figur durch Striche markiert. Sowohl die Abbildungen als auch der Hintergrund sind geriffelt - auf die Oberfläche des Steins sind enge und parallele Rillen eingehauen. Offensichtlich weist diese

12 Die Information ist nicht korrekt, dass das Kapitell sechseckig sei (Helmi Üprus, Raidkivikunst Eestis XIII - XVII sajandini, hrsg. von Voldemar Vaga (Tallinn: Kunst, 1987), 66). 


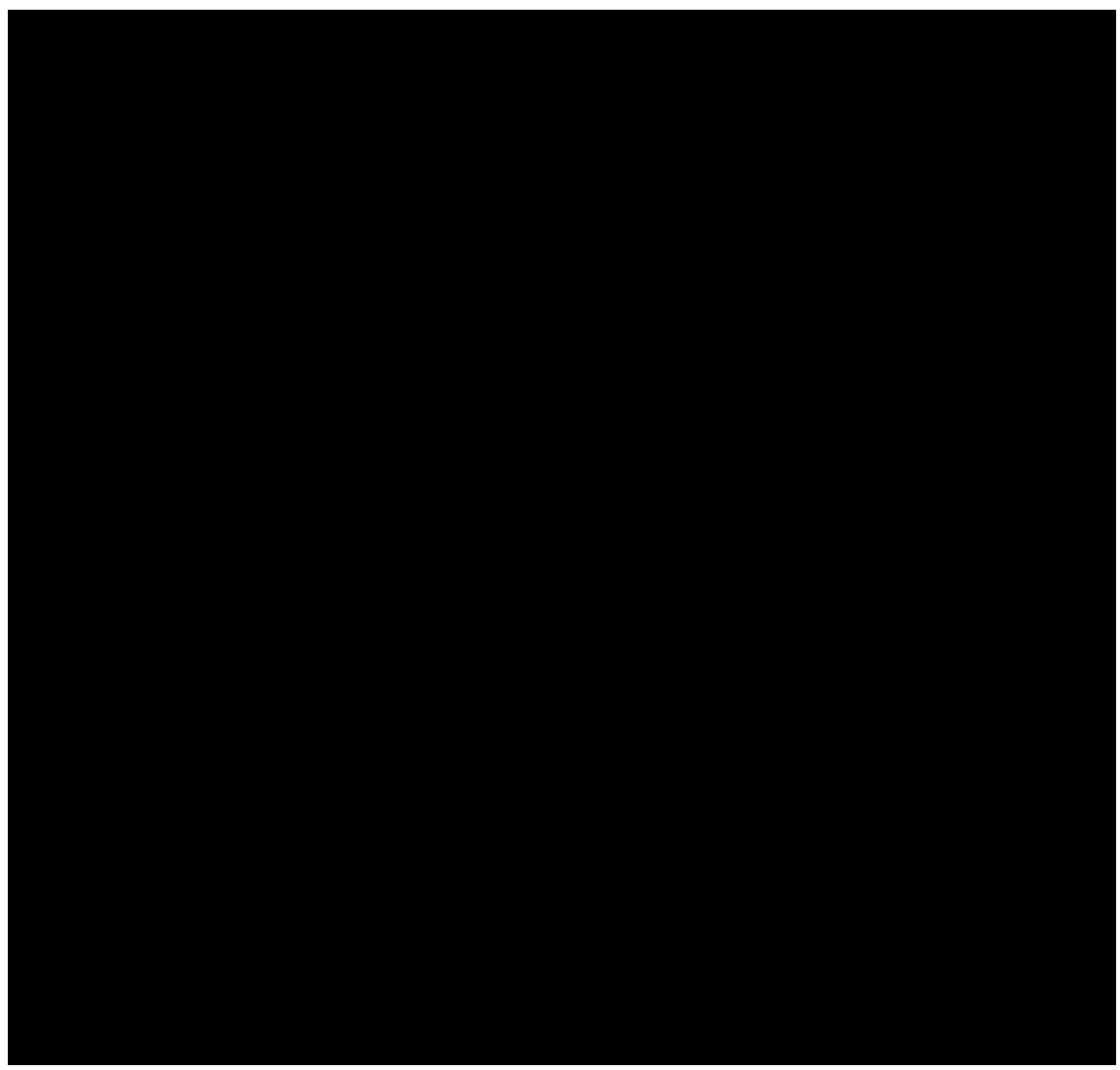

Abb. 4.-9. Details des Kapitells. Fotos von Kaur Alttoa.

Technologie auf Pläne hin, das Kapitell zu färben, denn Farbe haftet auf einer rauen Oberfläche besser.

Bei der Datierung des Kapitells und der gesamten Kapelle verwies Karl von Löwis of Menar auf zwei parallele Beispiele in Lettland: Die sich in Volmar (lett. Valmiera) befindliche St. Simonskirche mit achteckigen Pfeilern sowie die St. Johanniskirche zu Wenden (lett. Cēsis), auf deren Kämpfern der Innenportale ebenfalls flache Fabeltiere abgebildet sind. Beide wurden laut Information der Chronik von Hermann von Wartberg im Jahr 1283 gegründet ${ }^{13}$. Laut dem damaligen Verständnis sollte dieses Datum auch den Bau der gesamten Kirche bedeuten. Davon ausgehend

13 "Hermanni de Wartberge Chronicon Livoniae", Scriptores rerum Prussicarum II, hrsg. von Ernst Strehlke (Leipzig: Hirzel, 1865), 50. 


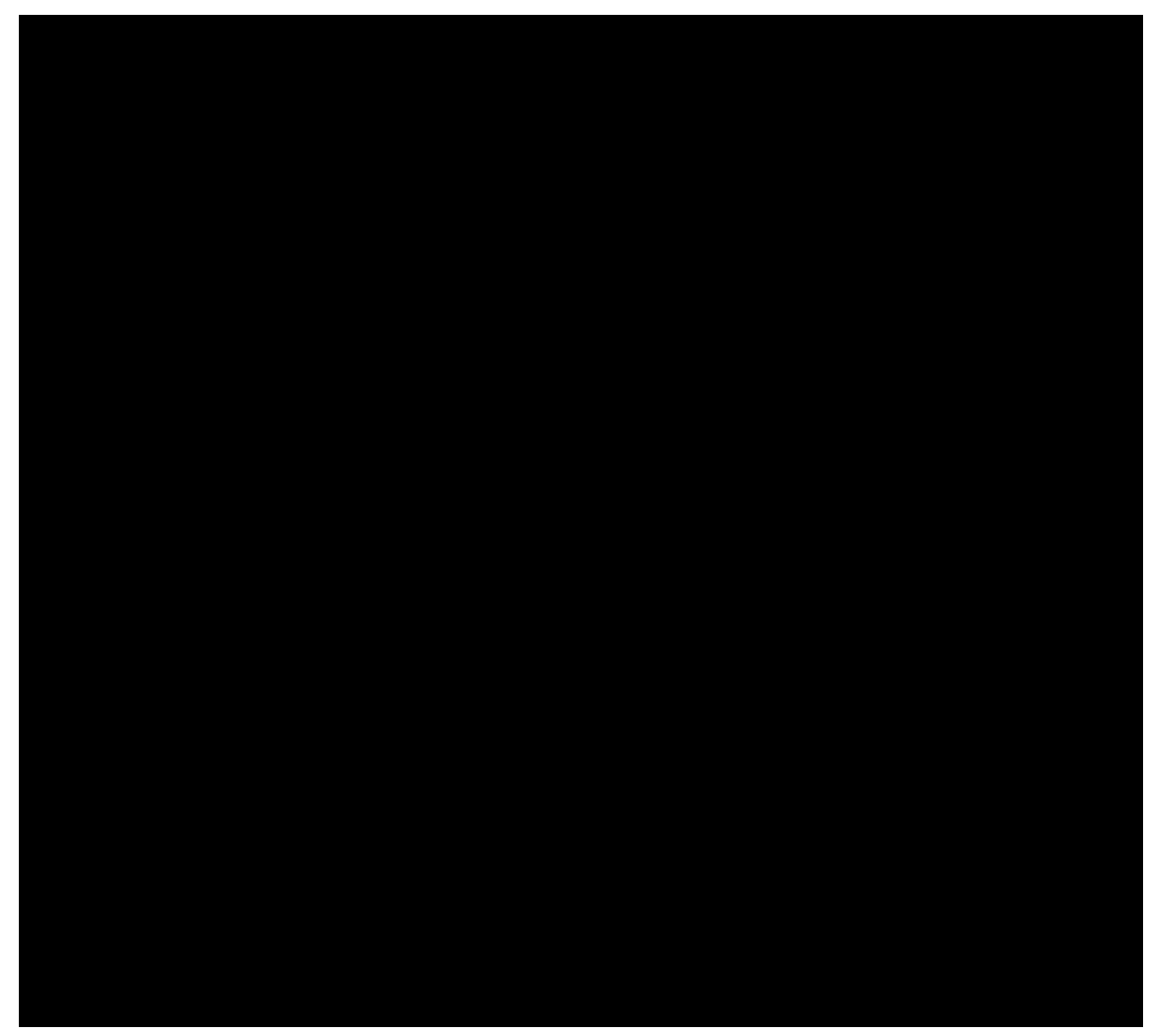

Abb. 10.-15. Details der Kapitelle aus dem Saal der Großen Gilde in Tallinn. Fotos von Kaur Alttoa.

verortete Karl von Löwis of Menar das Kapitell in Fellin in das letzte Viertel des 13. Jahrhunderts. ${ }^{14}$ Später hat Armin Tuulse das Kapitell behandelt.. ${ }^{15}$ Er verweist auf ein paralleles Beispiel, welches dem Kapitell aus Viljandi sehr ähnlich ist. Es handelt sich um die Pfeilerkapitelle des Saals der Großen Gilde in Tallinn (heute zum Estnischen Geschichtsmuseum gehörend, est. Eesti Ajaloomuuseum). Auch dort werden Adler, Fabeltiere und Pflanzenmotive abgebildet, alles im flachen, sogenannten Herbariumstil. Die Einzelelemente der Kapitelle in Tallinn und Viljandi sind sehr ähnlich geschnitten, vergleichbar ist auch die Riffelung der Oberfläche. Offenbar

14 Löwis of Menar, "Freilegung der Überbleibsel der St. Katharinenkapelle in Fellin”, 10.

15 Armin Tuulse, "Viljandi ordulossi kapiteelid", Litterarum Societas Esthonica 1838-1938. Liber Saecularis II. Commentationes Litterarum Societatis Esthonicae XXX (Tartu, 1938), 765; Armin Tuulse, "Die spätmittelalterliche Steinskulptur in Estland und Lettland", Suomen Muinasmuistoyhdistyksen Aikakauskirja, XLIX:1 (Helsinki, 1948), 37-39. 


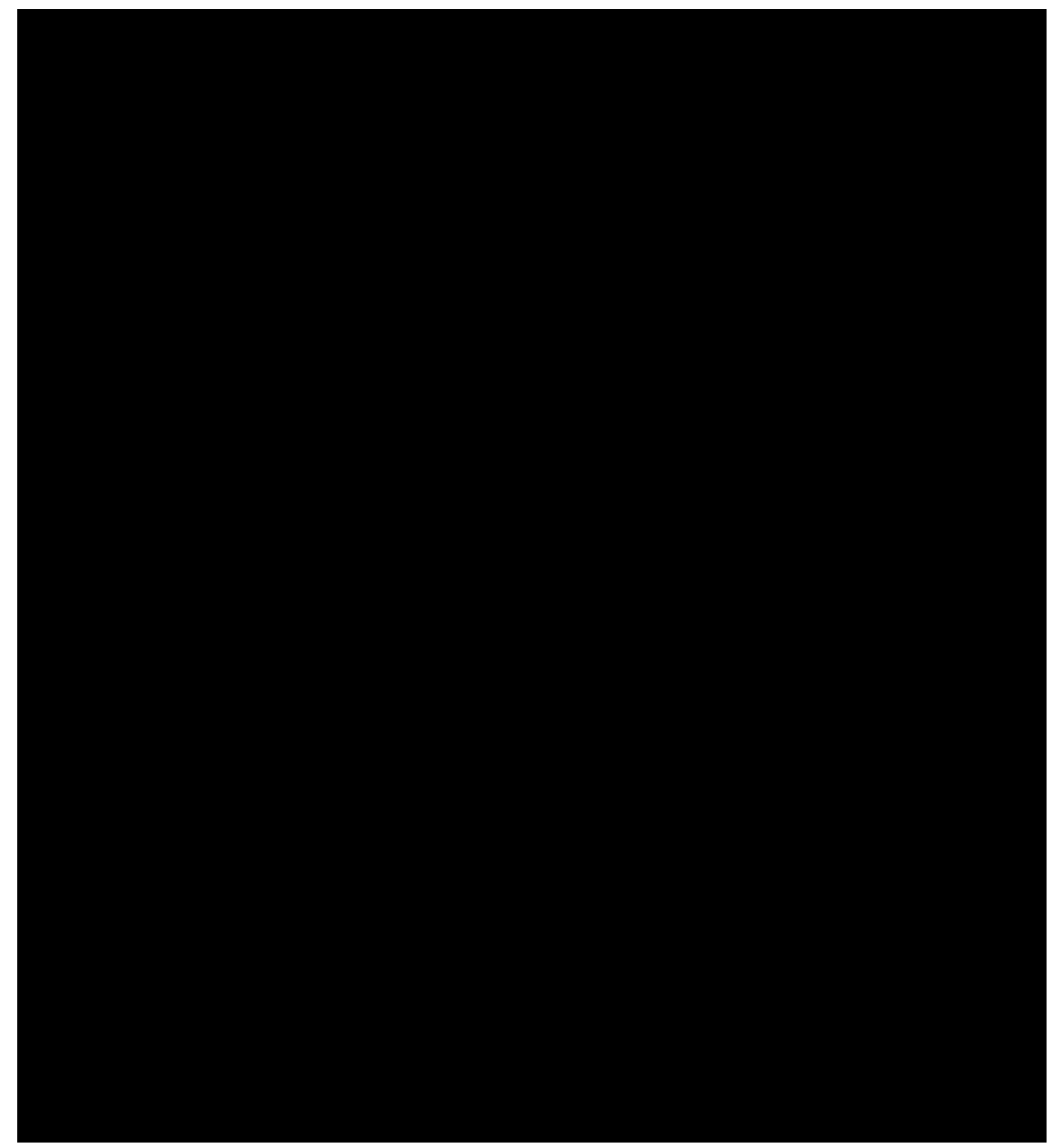

Abb. 16.-17. Kämpfer des Portals der St. Johanniskirche in Cēsis. Fotos von Kaur Alttoa.

handelte es sich um das Werk desselben Meisters. Die Kapitelle der Großen Gilde sind auch exakt datiert - einem ist die Jahreszahl MCCCCX eingeschnitten. Damit müsste auch das Kapitell in Viljandi aus dieser Zeit am Anfang des 15. Jahrhunderts stammen. Den Standpunkt Armin Tuulses hat auch Helmi Üprus unterstützt. ${ }^{16}$

16 Helmi Üprus, Raidkivikunst Eestis XIII - XVII sajandini, 66. 


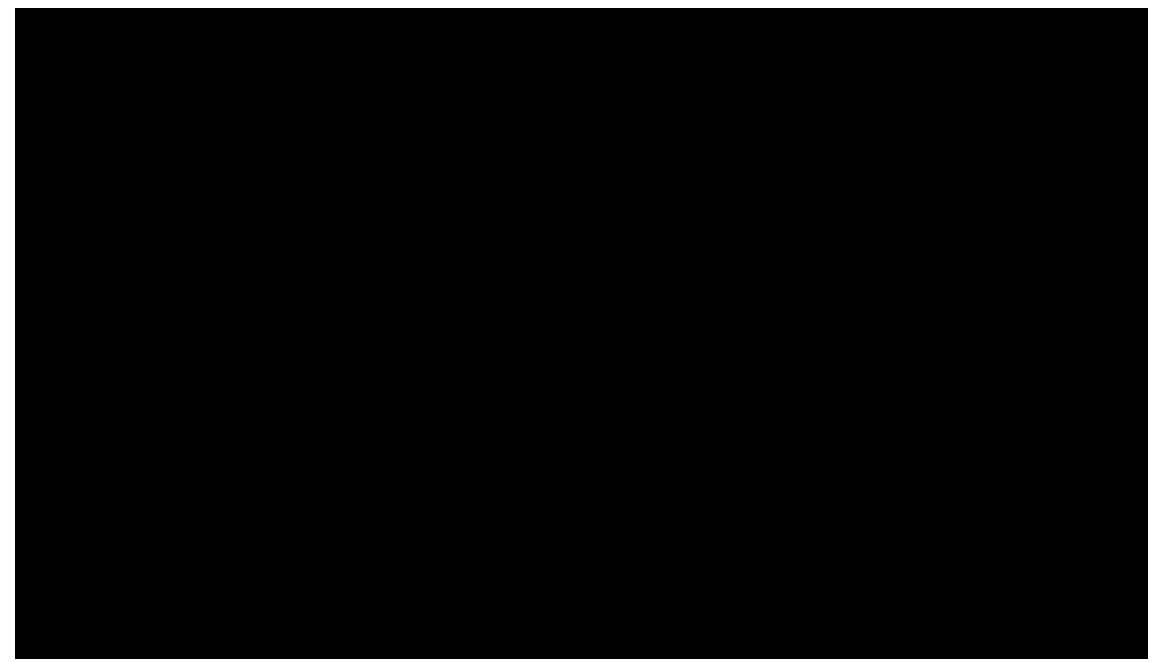

Abb. 18. Kämpfer eines Portals in Tallinn in der Vene tänav 12. Foto von Kaur Alttoa.

Tuulse ortet auch die Portalskulpturen der St. Johanniskirche in Cēsis in eine Reihe mit den Kapitellen in Tallinn und Viljandi ein. ${ }^{17}$ Seine Ansicht haben auch lettische Forscher geteilt. ${ }^{18}$ Die Kämpfer des Portals in Cēsis sind gerade und nicht gegliedert - eine Lösung, die sehr charakteristisch für Portale gerade in Tallinn ist. Auch die dargestellten Fabeltiere ähneln in mancher Hinsicht denen in Viljandi. Doch bestehen hier auch einige Unterschiede in der "Handschrift" des Meisters. So wurde hier versucht, einzelne Details, beispielsweise den Schwanz eines Fabeltieres, zu modellieren. Der größte Teil der Oberfläche der Figuren ist aber geriffelt. Linien zeigen deutlich, dass der Steinblock schon derartig bearbeitet wurde, bevor die Figuren eingeschnitten wurden. Verständlicherweise erscheint es als möglich, dass dieser technologische Unterschied durch das zu verwendende Material hervorgerufen wurde. Dennoch kann nicht mit ganzer Sicherheit behauptet werden, dass derselbe Meister die Kämpfer in Cēsis behauen hat, der auch die Kapitelle in Tallinn in der Großen Gilde und in Viljandi geschaffen hat. Zweifelsohne gehören sie aber alle in dieselbe Epoche. Wohl sollte angemerkt werden, dass wenigstens bei der Revaler

17 Tuulse, Die spätmittelalterliche Steinskulptur in Estland und Lettland, 39.

18 Jānis Zìlgavis, "St. John’s Church in Cesis”, Eiropa - kopīgs mantojums. Baznīcas Latvijā (Rīga: Valsts kultūras pieminekḷu aizsardzības inspekcija, 1999), Nr. 14; Andris Caune, Ieva Ose, Latvijas viduslaiku mūra baznīcas 12. gs. beigas - 16. gs. sākums. Enciklopēdija (Riga: Latvijas vēstures institūta apgāds, 2010), 118. 
Steinskulptur solche pfefferkuchenartig abgebildeten Figuren deutlich länger auftreten als nur am Anfang des 15. Jahrhunderts. Hier muss das Portal eines Kaufmannshauses (heute Stadtmuseum, est. Linnamuseum) in der Vene tänav 17 (dt. Russische Straße) erwähnt werden, auf dessen Kämpfern sich flache Löwenfiguren befinden. Es gibt aber Probleme mit der Datierung dieses Portals. Es ist behauptet worden, die Fassade des Gebäudes zusammen mit dem Portal stamme aus dem Jahr 1506 - denn dieses Datum findet sich auf einem Pfeiler des in der Diele befindlichen Mantelschorsteins..$^{19}$ In der Realität erscheint es als höchst fragwürdig, die Grundkonstruktion eines Hauses auf der Basis eines Gestaltungselementes des Interieurs zu datieren. Offensichtlich stammt die Fassade des Gebäudes mitsamt Portal dennoch aus dem 15. Jahrhundert - vermutlicherweise aber doch eher aus der zweiten Hälfte des Jahrhunderts. Somit ist ein solcher "Herbariumstil" in Reval für wenigstens ein halbes Jahrhundert verbreitet gewesen. Damit fehlt auch die Grundlage für eine genauere Datierung des Portals der St. Johanniskirche.

\section{DIE FRAGE NACH DER HERKUNFT DES KAPITELLS IN VILJANDI}

Alle Forscher - den Verfasser dieses Beitrags eingeschlossen ${ }^{20}$ - haben ohne zu zögern behauptet, dass dieses Kapitell einst die St. KatharinenKapelle in Fellin verschönert hatte. Dennoch kann man sich dessen nicht ganz sicher sein. Denn auf Basis des Grundrisses der Kapelle ist es eindeutig klar, dass hier nur ein Pfeiler gestanden haben konnte, bei den Ausgrabungen 1908 wurden aber ganze zwei Kapitelle gefunden. Das zweite $^{21}$ ist ebenfalls aus Kalkstein und achteckig. Dessen Seiten verschönern vertiefte Vierpasse, deren unterer Teil fehlt. Offensichtlich bestand das Kapitell aus zwei Blöcken. Ein zum Verwechseln ähnliches Kapitell, bei dem wieder der untere Teil des Dekors fehlt, wurde 1980 in der St. Johanniskirche in Viljandi aufgefunden, wo dieses im 19. Jahrhundert

19 Teddy Böckler, “Tallinna Linnamuuseum Vene t. 17”, Eesti Arhitektuur 1. Tallinn. Gesamtherausgeber Villem Raam (Tallinn: Valgus, 1993), 271. Die tatsächliche Jahreszahl lautet nämlich 1503. Für diese Richtigstellung dankt der Autor Anu Mänd.

20 Kaur Alttoa, "Katariina kabel”, Eesti Arhitektuur 2. Läänemaa, Saaremaa, Hiiumaa, Pärnumaa, Viljandimaa. Gesamtherausgeber Villem Raam (Tallinn: Valgus, 1996), 166.

21 Das Kapitell befindet sich im Museum in Viljandi. 


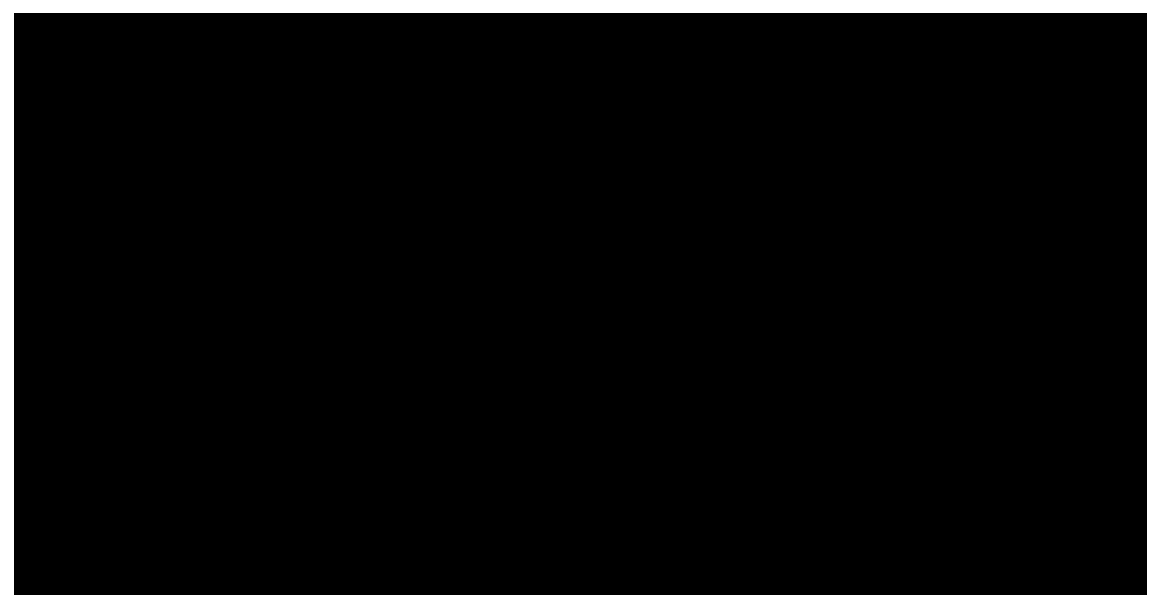

Abb. 19. Das Vierpass-Kapitell, gefunden auf dem Standort der Kapelle. Foto von Kaur Alttoa.

als Fundament des Fußes der Kanzel Verwendung gefunden hatte. ${ }^{22}$ Es ist klar, dass diese beiden Kapitelle ein Paar bildeten. Für sie fehlte der Raum in der St. Katharinen-Kapelle, damit befanden sie sich anfangs offenbar irgendwo anders. So mag vermutet werden, dass es sich um ein architektonisches Erbe des mittelalterlichen Fellin handelte, vielleicht einer damaligen Stadtkirche, das beim Abriss auf den Müll geriet mitsamt seiner Einzelteile, wovon eines in die St. Johanniskirche gelangte, das andere aber auf das ehemalige Gelände des Katharinen-Klosters.

Karl von Löwis of Menar erwähnt noch etwas, was bei der Ausgrabung gefunden wurde, ein plastisch gearbeitetes, eine Weintraube darstellendes Kalksteinstück, was nach Auffassung dieses Forschers aus der Eck- oder Wandkonsole der Kapelle stammte. ${ }^{23}$ Auf Grund der Behandlung des Weinlaubs wird es deutlich, dass es sich um denselben Meister handelt, der auch wenigstens ein Kapitell des Kreuzganges der Burg Fellin sowie ebenfalls auch das Südportal der Kirche in Turgel (est. Türi) geschaffen hat. Sie sind wahrscheinlich im letzten Viertel des 13. Jahrhundert ange-

22 Es handelte sich um eine ehemalige Klosterkirche der Franziskaner, welche im 17. Jahrhundert zu einer lutherischen Gemeindekirche der Stadt umgebaut wurde. Im Verlauf der Ausgrabungen wurde das Kapitell im Holzschuppen des Pastorats gelagert. Über den heutigen Standort des Kapitells fehlen jegliche Angaben.

23 Löwis of Menar, "Freilegung der Überbleibsel der St. Katharinenkapelle in Fellin", 8. Das Fragment der Steinskulptur ist im Speicher von Viljandi Folkmusik (est. Viljandi pärimusmuusika ait) ausgestellt. 


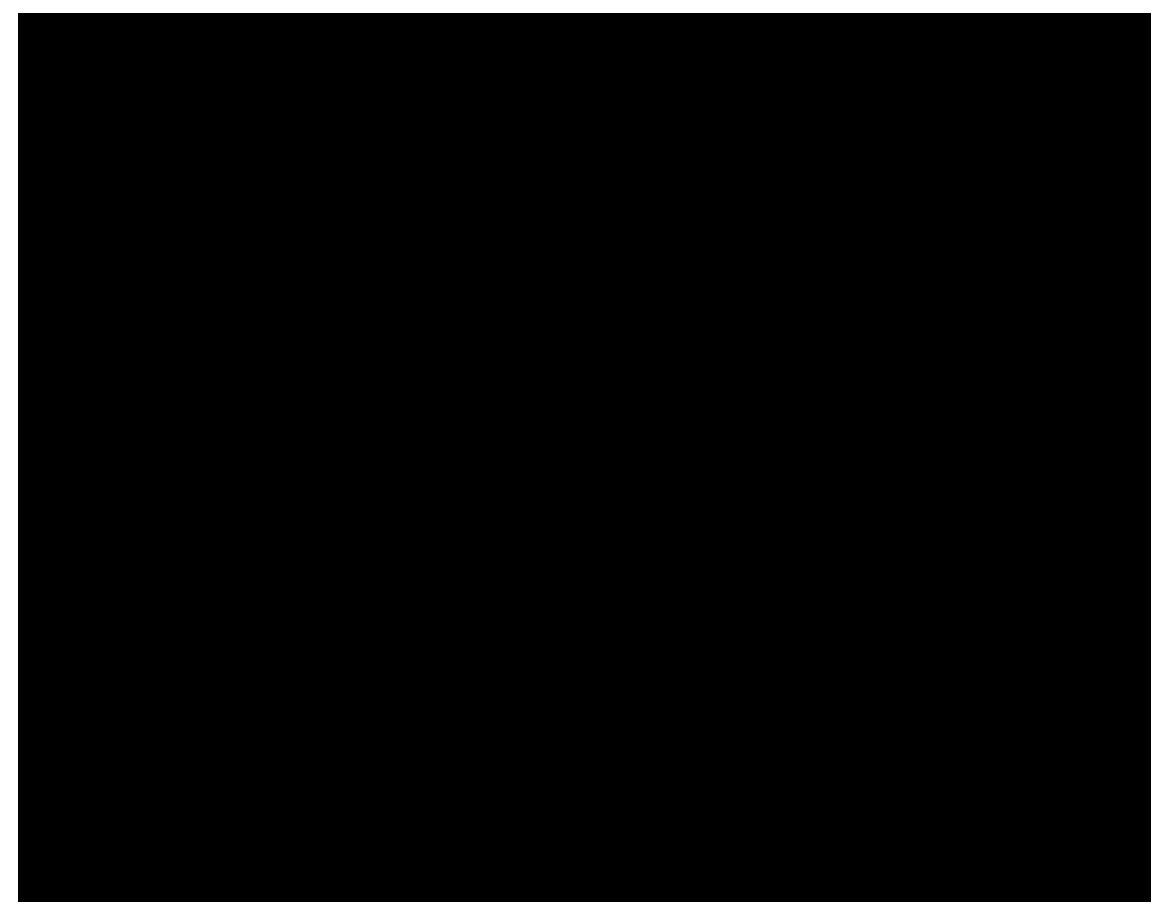

Abb. 20. Das in der St. Johanniskirche in Viljandi gefundene Kapitell. Foto von Kaur Alttoa.

fertigt worden oder spätestens um das Jahr 1300 herum. ${ }^{24}$ Zweifelsohne hat das zu untersuchende Fragment einer Steinskulptur nichts mit der St. Katharinen-Kapelle zu tun, sondern es stammte stattdessen aus der Burg. Somit wurden auf dem Gelände dieser Kapelle zwei Bestandteile von mittelalterlichen Steinskulpturen gefunden, die nicht aus der Kapelle stammten. Es scheint nicht als unmöglich, dass sie dann dorthin gelangten, als dies Gebiet zum Müllberg geworden war. In diesem Fall aber muss die Möglichkeit berücksichtigt werden, dass auch das Kapitell mit den Fabeltieren einen vergleichbaren Weg hinter sich hatte und dass es überhaupt nicht mit der Katharinen-Kapelle in Verbindung steht.

24 Kaur Alttoa, "Viljandi ordulinnuse arhitektuur. On the Architecture of Viljandi Castle", Viljandi ordulinnus ja lossimäed läbi aja. The Teutonic Order's Castle Hills in Viljandi Through Time. Viljandi Muuseumi toimetised V (Viljandi, 2015), 102. 


\section{GEWÖLBERIPPEN MIT DOPPELTEM WULST}

Das Kapitell mit den Fabeltieren ist der einzige Ausgangspunkt bei der Datierung der Katharinen-Kapelle In einer Situation, in welcher diese in Zweifel gezogen wird, folgt automatisch die Frage, wann denn die St. Katharinen-Kapelle tatsächlich gebaut wurde? Einige Informationen bieten die Rippensteine aus Ton. Diese wurden bei den Ausgrabungen in derart großer Zahl gefunden, dass es sich schwerlich um von andernorts hergebrachten Müll handeln konnte. Offensichtlich stammen sie vom Gewölbe der Katharinen-Kapelle selbst. Das Profil der Rippen besteht aus zwei bescheidenen Wülsten. Ungewöhnlich ist die Herstellungstechnologie der Rippensteine. Sie wurden nicht im Formkasten gefertigt, sondern mit Hilfe einer Schablone aus Lehmblöcken mit der Dicke von Ziegelsteinen als Rohling ausgeschnitten. ${ }^{25}$ Rippensteine mit dem erwähnten Profil sind in Südestland weit verbreitet: Neben der Katharina-Kapelle in Fellin sind sie wenigstens an vier weiteren Orten bekannt. Wohl bestehen Probleme mit ihrer exakten Datierung. Diese Form wurde in der Kirche von Randem (est. Rannu) genutzt, welche vermutlich im zweiten Viertel des 15. Jahrhunderts errichtet wurde. ${ }^{26}$ Ähnliche Rippensteine waren auch in den Kapellen der Westtürme der Domkirche zu Dorpat eingesetzt worden. Die Türme wurden an der Wende vom 15. auf das 16. Jahrhundert fertiggestellt, die Kapellen wurden aber etwa später hinzugefügt. Ihre zeitliche Grenze ante quem sollte aber das Jahr 1525 darstellen - nach dem Durchbruch der Reformation in Livland erscheint eine Bautätigkeit von Seiten des katholischen Bischofs mehr als fragwürdig. ${ }^{27}$ Eine gleiche Form der Rippen findet sich auch in der Sakristei der St. Johanniskirche in Dorpat ${ }^{28}$ und ein einzelner Ziegelstein in solcher Form wurde auch in der Kirche von Kamby (est. Kambja) gefunden. ${ }^{29}$ In beiden Fällen fehlen Bezugspunkte für ihre Datierung. In Riga befinden sich solche Rippenprofile im 1407 begonnenen Gewölbe des Chorumgangs der Petrikirche und Stücke derartiger

25 Eine ebensolche Technik wurde auch bei den Rippen der Sakristei der St. Johanniskirche in Dorpat angewendet.

26 Kaur Alttoa, "Märkmeid Lõuna-Eesti keskaegsetest maakirikutest", Ars Estoniae medii aevi. Grates Villem Raam, Viro Doctissimo et Expertissimo (Tallinn: Eesti Muinsuskaitse Selts, 1995), 73.

27 Kaur Alttoa, "Tartu toomkiriku läänetornide kaitsefunktsioonist", Arhitektuuripärandi uurimisest ENSV-s (Tallinn: Valgus, 1987), 87.

28 Kaur Alttoa, Tartu Jaani kirik. Mitautoren Eve Alttoa, Krista Kodres und Anu Mänd. Eesti kirikud III (Tallinn: Muinsuskaitseamet, 2011), 22.

29 Kaur Alttoa, "Keskaegsest Kambja kirikust", Wastne testament 1696 (Tartu: B. G. Forseliuse Selts, 1996), 78. 


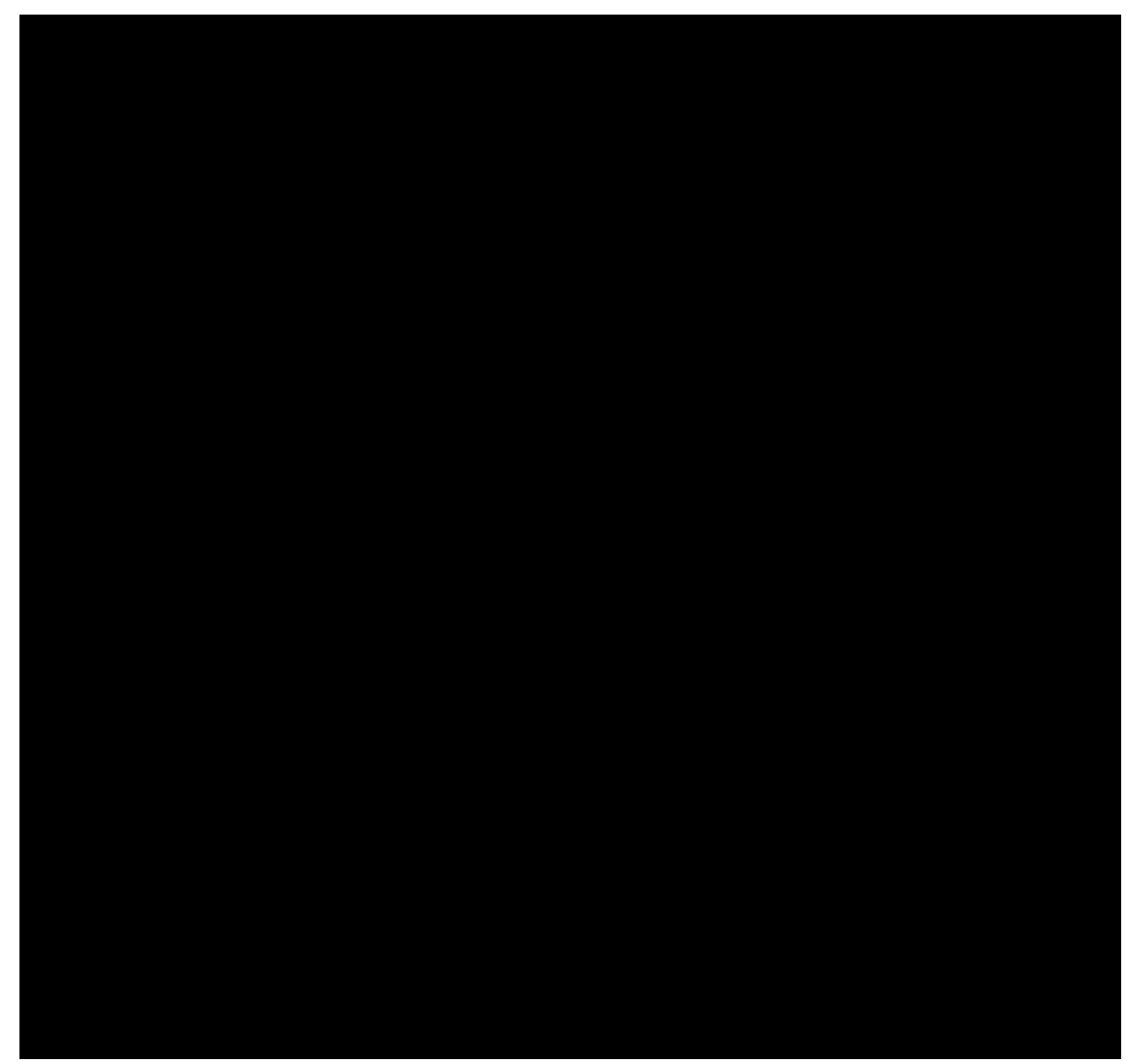

Abb. 21. Das skulpturierte Fragment, welches auf dem Ort der St. Katharina-Kapelle aufgefunden wurde. Foto von Kaur Alttoa.

Formsteine wurden auch für die Pfeiler als Mauermaterial genutzt ${ }^{30}-$ sie müssten demnach auf jeden Fall aus dem ersten oder zweiten Jahrzehnt des 15. Jahrhunderts stammen. Ebensolche Rippenform trifft man auch in den Seitenkapellen der Domkirche in Riga an. Damit sind Rippen in der zu betrachtenden Form auf dem Backsteingebiet Altlivlands für lange Zeit, durch das 15. Jahrhundert hindurch und auch in den ersten Jahrzehnten des folgenden Jahrhunderts, in Gebrauch gewesen. ${ }^{31}$ Auf dieser Grundlage

30 Gunārs Zirnis, Pētera baznīca (Rīga: Zinātne, 1984), 62-63.

31 Es sei noch ergänzt, dass ähnliche Rippenziegelsteine auch 1433 beim Bau des Novgoroder Facettenpalasts (russ. Granovitaja Palata) verwendet worden sind (Ilya Antipov, Dmitry Yakovlev, "The Faceted Palace in Novgorod the Great as the part of the Archbishop's residence", Castella Maris Baltici XII. Castle as a Residence (Łódź: Institute of Archaeology, University of Łódź), 109-111). 


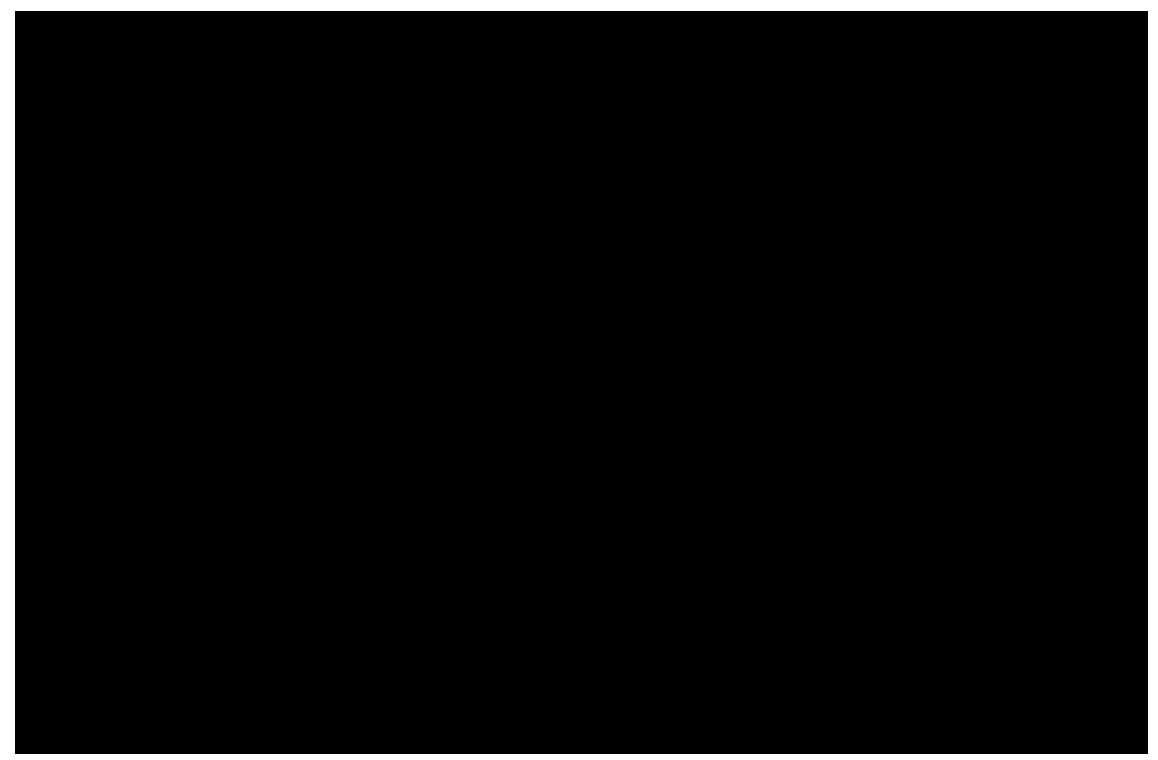

Abb. 22. Die Rippensteine der St. Katharinen-Kapelle im Museum in Viljandi. Foto von Kaur Alttoa.

erscheint es als glaubhaft, dass die St. Katharinen-Kapelle in Fellin im 15. Jahrhundert erbaut wurde. Etwas Genaueres lässt sich leider nicht sagen.

\section{FAZIT}

Außerhalb der Stadtmauern Fellins befand sich die St. KatharinenKapelle. Diese wurde im 15. Jahrhundert gebaut, exaktere Ausgangsdaten zur Datierung fehlen. Das auf dem Gebiet der Kapelle gefundene Kapitell mit Reliefs wurde offenbar am Anfang des 15. Jahrhunderts fertiggestellt. Doch es besteht keine Sicherheit, dass dieses Kapitell auch aus der Kapelle in Fellin stammt. 
Kaur Alttoa: Viljandi's St. Catherine's Chapel

Keywords: Viljandi; Medieval Chapel; Late Gothic Stone Carving; House of the Great Guild in Tallinn; St. John's Church in Cēsis

\section{SUMMARY:}

During the Middle Ages, St. Catherine's Chapel in Viljandi was located outside the town walls, and it was demolished in 1558, upon the outbreak of the Livonian War. In 1908, archaeological excavations were conducted in the vicinity of the former chapel under the direction of Karl Löwis of Menar. An octagonal limestone capital (now located in the Viljandi Museum) was found in the course of the excavations. Its facets are decorated with slightly raised fantastical animals and plant motifs. The capitals in the Great Guild Hall (currently the Estonian History Museum) in Tallinn that date from 1410 are very similar to the Viljandi capital. Apparently, they are the work of the same master. Therefore, we can date the Viljandi capital to the early $15^{\text {th }}$ century. However, other sculptural medieval elements, which are not related to St. Catherine's Chapel, have also been found in its vicinity. Apparently, they have ended up there with the waste from subsequent centuries. So, we cannot say with certainty whether the capital with the relief decorations is from St. Catherine's Chapel.

A large number of brick rib stones have also been found in the vicinity of the chapel. A similar form was in wide use in Livonia (Southern Estonia and also Riga) throughout the $15^{\text {th }}$ century. Apparently, Viljandi's St. Catherine's Chapel was also built at that time. With the research that has been conducted to date, it is not possible to be sure about the dating of the chapel.

CV:

Kaur Alttoa has been a long-time scholar and teacher of art history at the University of Tartu and he is one of the foremost specialists of the history of medieval architecture in the Baltic region. His objects of interest have included both medieval fortresses and sacral buildings. Among his major contributions stands out the restoration and study of the St. John's Church in Tartu, which was also published as a monograph in 2011. 